EPJ manuscript No.

(will be inserted by the editor)

\title{
Spin-dependent transport in cluster-assembled nanostructures: influence of cluster size and matrix material
}

\author{
Matthias Hillenkamp ${ }^{1}$, Giulia di Domenicantonio ${ }^{1}$, Christian Félix ${ }^{1}$, \\ Laurent Gravier ${ }^{2}$, Santiago Serrano-Guisan ${ }^{2}$ and Jean-Philippe Ansermet ${ }^{2}$ \\ 1 Laboratoire des Nanostructures en Matrices, Ecole Polytechnique Fédérale de Lausanne, CH-1015 Lausanne-EPFL, Switzer- \\ land \\ 2 Laboratoire de Physique des Matériaux Nanostructurés, Ecole Polytechnique Fédérale de Lausanne, CH-1015 Lausanne-EPFL, \\ Switzerland
}

Received: date / Revised version: date

\begin{abstract}
Spin-dependent transport in granular metallic nanostructures has been investigated by means of a thermoelectric measurement. Cobalt clusters of well-defined size $(\langle n\rangle=15-600)$ embedded in copper and silver matrices show magnetic field responses of up to several hundred percent at low temperature. The experimental observations are identified as due to spin mixing. The influence of cluster size and matrix are discussed.
\end{abstract}

PACS. 72.25.Rb Spin relaxation and scattering - 73.63.-b Electronic transport in nanoscale materials and structures

\section{Introduction}

The field of spintronics has attracted a lot of attention in the last few years due to its proposed enhanced device functionality. Generally, it deals with the interaction between the magnetization of the sample and the different conduction electron spin channels. One can roughly distinguish two approaches: the effect of the magnetic field on the different spin channels (as in giant and tunnel magnetoresistance: GMR [1,2], TMR[3]) and, inversely, the effect of a polarized current on the local magnetization as observed in spin transfer torque experiments [4]. In particular the interest in magnetic nanoparticles has increased in the past few years by virtue of their potential application in fields such as ultrahigh-density recording and medicine. The ongoing miniaturization of magnetic storage devices has reached dimensions of $<100 \mathrm{~nm}$, a regime where intrinsic quantum mechanical effects and superparamagnetism become important [5]. Granular systems have already been proven to show spin-dependent effects like GMR in the early 90's [6,7], in later years the deposition of pre-formed clusters in the nm-range in matrices allowed the preparation of better defined samples and first investigations on the cluster size dependency of GMR [8].

However, no elastic interaction between the two spin channels is normally taken account of, albeit its consequence of reducing GMR [9]. We have developed a specific thermoelectric measurement protocol that permits to suppress the dominant part of the resistivity and allows us to extract temperature dependent effects that cannot be ex- plained by an uncoupled spin-dependent transport [10, 11]. In order to demonstrate the importance of spin-mixing processes in nano-structured systems we have fabricated a series of samples consisting of different well-defined Cobalt cluster sizes embedded in Copper and Silver matrices. Thus we show that the high sensitivity of the thermoelectric signal found with $\mathrm{Cu}$ [10] occurs also with $\mathrm{Ag}$ as a matrix. This signal is not correlated to spin-dependent transport properties like GMR and therefore must arise from another mechanism of the conduction electron spin dynamics.

\section{Experimental}

Samples are prepared according to the strategy of "clusterassembled materials" [12]. They consist of thin films of copper or silver in which are dispersed well-defined cobalt clusters. Narrow distributions of metal cluster ions are prepared and analyzed in the gas phase and co-deposited with the metal matrix on a substrate of slightly conductive polyimide (see Fig. 1). This method allows for the simultaneous control of the cluster size and their concentration. The film thickness is $50 \mathrm{~nm}$. We perform our measurements on stripes $<1 \mathrm{~mm}$ wide and $10 \mathrm{~mm}$ long, connected with silver paste to the electrodes.

Besides magneto-resistance measurements, we carried out a thermoelectric experiment that measures the thermogalvanic voltage (TGV) and was initially developed for multilayer systems [11]. The principle of this experiment is shown in Fig. 2: a chopped laser beam irradiates the film 


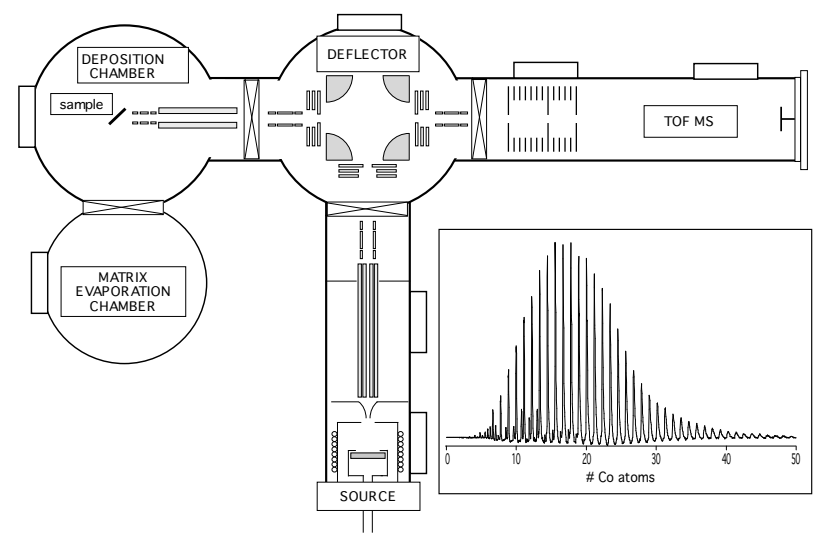

Fig. 1. Schematic overview of the experimental setup used to produce the cluster assembled samples. the insert shows a typical mass spectrum of Co cluster ions.

and induces a temperature oscillation of about $1 \mathrm{~K}$. The laser irradiates the center of the film and does not induce a net temperature gradient. The corresponding voltage across the whole film is measured by lock-in detection under a constant charge current $I_{D C}$. Although there could also be a contribution from the derivative of the resistance with respect to temperature, this effect, however, is negligible in metals below $\sim 20 \mathrm{~K}$, where the resistance is temperature independent. Consequently, TGV measurements carried out at $13-14 \mathrm{~K}$ are independent of the temperature dependence of the resistance. Variation of the external magnetic field yields the magneto-thermogalvanic voltage (MTGV).

\section{Results and Discussion}

MTGV measurements were carried out on samples with two different cluster sizes embedded in two different matrix materials, respectively. Figs. $3 \mathrm{a}$ and $\mathrm{b}$ reproduce the data for $\mathrm{Co}$ clusters in $\mathrm{Cu}$ matrices [10], Figs. 3c and d show data for Co clusters embedded in Ag. Although the matrix metal is different and also parameters like concentration and size differ, the general trends are well reproduced. Small clusters show very large MTGV responses of several $100 \%$ and are far from saturation. The signal of

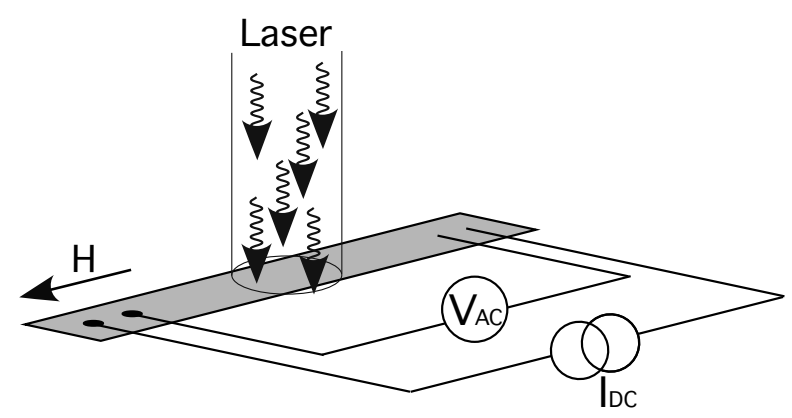

Fig. 2. TGV experiment: The voltage over the sample through which passes a constant current $I_{D C}$ is detected in phase with the temperature oscillation induced by chopped laser light.

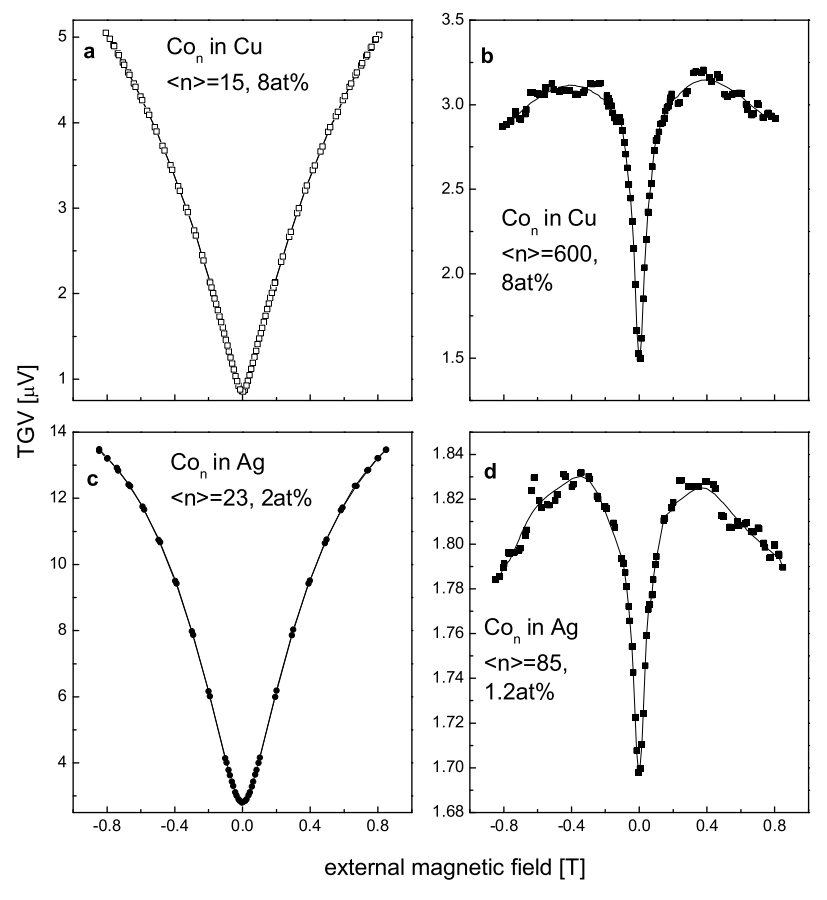

Fig. 3. MTGV curves for Co clusters in $\mathrm{Cu}$ and $\mathrm{Ag}$ matrices. Mean cluster sizes and atomic percentages are as noted in the figures. Solid lines are spline fits to guide the eye.

larger clusters is considerably smaller and saturates at low fields before slowly decreasing again. Conventional magnetoresistance measurements on all samples yield signals in the percent range or below with shapes differing from the MTGV curves [10].

Following the thermodynamic argument in Ref. [10] we can identify the different contributions to the MTGV signal. The thermogalvanic experiment measures the first derivative of the effective conductivity with respect to the temperature and comprises two main terms:

- The spin-dependent conductivities describe the uncoupled spin channels. They depend on the relative orientation of the magnetic grains, i.e. the misalignment of successive grains and are responsible for the GMR.

- An additional term stands for effects coupling the two spin channels, i.e. spin mixing. Differences between GMR and MTGV in their field and temperature dependence are due to this term.

Since the magnetoresistive responses of all samples discussed here clearly differ in both magnitude as well as shape from the MTGV signals shown, we infer spin mixing as responsible for this difference.

Spin mixing has previously been invoked in multi-layer systems, where electron-magnon collisions are proposed responsible for spin-flip scattering. However, given the size of the Co clusters considered in this study, scattering of conduction electrons by low-q magnons seems unlikely [13] and another spin-mixing mechanism must be considered. 
In granular systems, where the grain size is much smaller than the spin diffusion length, the spins of the conduction electrons precess about the exchange field as they traverse the magnetic cluster. This mechanism was named the "jitterbug spin channel mixing" [9] and effectively couples the two channels if two adjacent moments are misaligned. The conduction electron spin transition are given according to Rabi's formula:

$$
P_{12}(t)=\sin ^{2} \theta \sin ^{2}\left(\frac{\Delta E}{2 \hbar} t\right)
$$

where $\theta$ denotes the angle between adjacent magnetic grains. At zero field the cluster magnetic moments are oriented arbitrarily in space and the jitterbug spin mixing is completely symmetric. As the external field preferentially orients the magnetic moments, an asymmetry of the respective rates between the two channels develops and consequently the MTGV signal increases. The successive orientation of cluster moments with increasing field thus explains on a qualitative level both the increase as well as the successive decrease of the observed MTGV signals.

An asymmetry of the spin mixing implies a local polarization of the conduction electrons. This is in accordance with the spin polarization invoked in order to explain the anomalous low temperature increase of magnetization as observed in extraordinary Hall effect measurements of granular samples [14].

Since the magneto-thermogalvanic experiment detects spin mixing asymmetries, it does not directly measure transport properties in the non-magnetic matrix. In the light of these considerations it is not too surprising to find comparable MTGV results for Co clusters in different metallic matrices. Undoped matrices show a thermogalvanic response, most probably due to interface effects between matrix and contacts or substrate. No magnetic field effect, however, could be detected. The matrix, on the other hand, does intervene indirectly in the MTGV experiments since the magnitude of the cluster magnetic moment strongly depends on the surrounding medium [15, 16]. We believe the different degree of quenching of the cluster magnetic moment [17] to be responsible for the difference in cluster size showing a comparable MTGV for Co-doped samples of $\mathrm{Cu}$ or $\mathrm{Ag}$ matrices.

Further experiments at lower temperature and higher fields are under way and are expected to show saturation also for small clusters. The application of a recently developed theoretical model [18] also promises further insight into the underlying physics of spin-dependent transport, spin mixing and its detection in thermoelectric experiments. indirect influence of the surrounding matrix are rationalized within a phenomenological model.

\section{References}

1. M. N. Baibich, J. M. Broto, A. Fert, F. N. Van Dau, F. Petroff, P. Eitenne, G. Creuzet, A. Friederich, and J. Chazelas, Phys. Rev. Lett. 61, 2472 (1988).

2. G. Binasch, P. Grünberg, F. Saurenbach, and W. Zinn, Phys. Rev. B 39, 4828 (1989).

3. J. S. Moodera, L. R. Kinder, T. M. Wong, and R. Meservey, Phys. Rev. Lett. 74, 3273 (1995).

4. A. A. Tulapurkar, Y. Suzuki, A. Fukushima, H. Kubota, H. Maehara, K. Tsunekawa, D. D. Djayaprawira, N. Watanabe, and S. Yuasa, Nature 339 (2005).

5. V. Skumryev, S. Stoyanov, Y. Zhang, G. Hadjipanayis, D. Givord, and J. Nogués, Nature 423, 850 (2003).

6. A. Berkowitz, J. Mitchell, M. Carey, A. Young, S. Zhang, F. Spada, F. Parker, A. Hutten, and G. Thomas, Phys. Rev. Lett. 68, 3745 (1992).

7. J. Q. Xiao, J. S. Jiang, and C. L. Chien, Phys. Rev. B 46, 9266 (1992).

8. F. Parent, J. Tuaillon, L. B. Stern, V. Dupuis, B. Prevel, A. Perez, P. Melinon, G. Guiraud, R. Morel, A. Barthélémy, and A. Fert, Phys. Rev. B 55, 3683 (1997).

9. J. F. Gregg, W. Allen, S. M. Thompson, M. L. Watson, and G. A. Gehring, J. Appl. Phys. 79, 5593 (1996).

10. S. Serrano-Guisan, G. di Domenicantonio, M. Abid, J.-P. Abid, M. Hillenkamp, L. Gravier, J.-P. Ansermet, and C. Félix, Nat. Mater. 5, 730 (2006).

11. L. Gravier, S. Serrano-Guisan, F. Reuse, and J.-P. Ansermet, Phys. Rev. B 73, 024419 (2006).

12. M. Hillenkamp, G. di Domenicantonio, and C. Félix, Rev. Sci. Instrum. 77, 025104 (2006).

13. L. Piraux, M. Cassart, J. S. Jiang, J. Q. Xiao, and C. L. Chien, Phys. Rev. B 48, 638 (1993).

14. A. Gerber, A. Milner, M. Karpovski, A. Tsukernik, A. Sulpice, J. Tuaillon-Combes, P. Melinon, and A. Perez, Phys. Rev. B 69, 134422 (2004).

15. C. Binns, K. N. Trohidou, J. Bansmann, S. H. Baker, J. A. Blackman, J.-P. Bucher, D. Kechrakos, A. Kleibert, S. Louch, K.-H. Meiwes-Broer, G. M. Pastor, A. Perez, and Y. Xie, J. Phys. D: Appl. Phys. 38, R357 (2005).

16. R. N. Nogueira and H. M. Petrilli, Phys. Rev. B 63, 012405 (2000).

17. D. Eastham, Y. Qiang, T. Maddock, J. Kraft, J.-P. Schille, G. Thompson, and H. Haberland, J. Phys. Condens. Matter 9, L497 (1997).

18. O. Tsyplyatyev, O. Kashuba, and V. I. Fal'ko, http://arxiv.org/abs/cond-mat/0609012.

\section{Conclusions}

Spin-dependent transport in metallic nanostructures has been studied using a magneto-thermogalvanic measurement. The observed phenomena are explained as due to spin mixing caused by the precession of the conduction electron spin about the cluster magnetic moments. The saturation behavior for different cluster sizes as well the 